\title{
The Lived Experience of Nurses Working in Cardiology Services with Online Continuing Professional Programs in Advancing their Specialized Clinical Practice: Phenomenology Study Methodology
}

Umaima Al-Raisi ${ }^{1}$, Issa Al Salmi ${ }^{2,3 *}$, Judy Magarey ${ }^{4}$, Philippa Rasmussen ${ }^{4}$, Suad Hannawi ${ }^{5}$

${ }^{1}$ National Heart Centre, the Royal Hospital, Muscat, Oman

${ }^{2}$ Medicine Department, the Royal Hospital, Muscat, Oman

${ }^{3}$ Medicine Department, Oman Medical Specialty Board, Muscat, Oman

${ }^{4}$ School of Nursing, the University of Adelaide, South Australia, Australia

${ }^{5}$ Medicine Department, Ministry of Health and Prevention (MOHAP), Dubai, UAE.

*Corresponding Author: Issa Al Salmi, Medicine Department, the Royal Hospital, Muscat, Oman, Oman Medical Specialty Board, Muscat, Oman.

Received Date: October 30, 2020; Accepted Date: November 13, 2020; Published Date: January 05, 2021

Citation: Umaima A. Raisi., Issa A. Salmi., Judy Magarey., Philippa Rasmussen., Suad Hannawi., (2021) The Lived Experience of Nurses Working in Cardiology Services with Online Continuing Professional Programs in Advancing their Specialized Clinical Practice: Phenomenology Study Methodology. J. Clinical Cardiology and Cardiovascular Interventions, 4(2); Doi:10.31579/2641-0419/102

Copyright: (c) 2021 Issa Al Salmi, This is an open-access article distributed under the terms of the Creative Commons Attribution License, which permits unrestricted use, distribution, and reproduction in any medium, provided the original author and source are credited.

\section{Abstract}

Introduction: Nurses should be committed to undertake continuing professional development (CPD) courses to advance nursing practice and guarantee lifelong learning. Online CPD programs may allow nurses to fulfil the demand for specialty competency.

Aim: This study focuses on utilising online (CPD) activities to develop cardiac nurses' ability to perform advanced clinical skills.

\begin{abstract}
Method: The study was conducted in one of the largest accredited teaching hospitals in South Australia. The department is staffed by a specialised multi-professional team, some of whom have completed specialised cardiac post-graduation diploma courses in order to meet the complex needs of cardiac patients. To keep the team abreast of the latest developments in practice, the in-service education department at local study setting runs several CPD programs for the cardiology department via varied learning modes, such as online CPD programs, classroom learning and bedside-based learning. The nursing team maintains advanced clinical skills through online CPD, orientation programs, and in-service classroom-based courses. Regarding online CPD courses, electrocardiography interpretation and underwater sealing draining management courses are mandatory courses which all registered nurses must complete while working in medical or surgical cardiac wards.
\end{abstract}

Results: The interview process was conducted in five stages: 1. Determining the type of the interview where in such types of qualitative studies the researcher should focus on the fundamental question of the phenomenological inquiry throughout the unstructured, in-depth interview process. 2. Making initial contact where the researcher established a rapport with the participant and prepared them mentally by giving them the participant information sheet. 3 . Context of the interview where interviews be conducted in a quiet room in the School of Nursing in order to maintain participant privacy and anonymity, participants requested to conduct the interviews in their work setting. Nonetheless, the researcher ensured that participant privacy and anonymity was upheld. 4. Selecting the lived experience where Each participant was interviewed once. Interview duration was 15 to 30 minutes. The interviews started with a grand tour question. Grand tour questions are very broad questions asked by the interviewer at the early stage of an interview to obtain a description of the event or experience. 5. All interviews were concluded by thanking the participant and offering them the choice to have a copy of their interview transcript to verify what they had said. The researcher wrote an interview summary after listening to the interviews on the same day. The summary was prepared to help the researcher evaluate the amount of data gathered and identify whether the point of data saturation was reached. In addition, writing the summary helped the researcher reflect on the interview and gain an understanding of the participant experience

Conclusion: This study explained the process of data collection, describing the setting, nature of participants and process of data collection using phenomenological interview. As the human experience is complex, gathering in-depth data should be systematic to ensure that the researcher has obtained the most sufficient data to explore the essence of the experience.

Keywords: cardiology; clinical practice; phenomenology; continuing professional development 


\section{Introduction}

Any health system depends on its daily basis upon the staff that carry the health care towards our patients. Health system empowers its people to deliver the care they wish to establish. Nurses are the corner of the health care system worldwide. Hence, preparation of a manual of professional ethics, development of a career structure for nurses, setting standards of nursing education; nursing practice, and issuing guidelines for registration and licensing would further enhance the population health perspectives [1-4].

The specialised clinical environment is very challenging and complex for medical staff. Those working in such an environment need to constantly update their knowledge and skills in order to be proficient in their specialty. It is known that nurses should be committed to undertake continuing professional development (CPD) courses to advance nursing practice and guarantee lifelong learning [5-7]. Online CPD programs may allow nurses to fulfil the demand for specialty competency [8]. Various adult learner needs, and preferred learning style are a concern for clinical educators and online CPD could provide knowledge acquisition to enable nurses to achieve a proficient level of competency to work in a specialised clinical setting [9]. Nurse experience using online CPD provides an insight regarding this method of learning.

Cardiology nursing is one of the most challenging nursing specialties. The complex needs of cardiac patients, increasing acuity of care and rapid evolution in medical, surgical and interventional cardiology imposes high demands on this branch of nursing [10]. Furthermore, the nature of the specialty drives the nurses' role to expand to deliver efficient, quality and cost-effective care. In other words, nurses are required to possess the competencies that aid them in providing rationalised, appropriate and effective interventions [10]. Riley, Brodie [11] emphasised that cardiac nurses should have the knowledge and skills to guide them not to only detect abnormalities, but also to diagnose and manage patients independently. Nurses should develop knowledge and skills pertaining to the varied and complex needs of their patients.

In the cardiac nursing context, there are three levels of clinical skills: core clinical skills, advanced clinical skills and specialised clinical skills [12]. Core clinical skills include basic, general nursing care; these skills are gained from undergraduate nursing education. Advanced clinical skills are those required to care for unstable patients who demand a high acuity of care. Specialised clinical skills require nurses to assess and manage complex patient needs and assist in interventional procedures [12]. Riley, Brodie [11] note that nurses need a post-registration education to attain specialist skills, whereas advanced clinical skills can be achieved via varied learning modes, such as in-service education activities, continuing professional development and orientation programmes.

This study focuses on utilising online (CPD) activities to develop cardiac nurses' ability to perform advanced clinical skills. To define advanced clinical skills referred to in this study, the researcher used Christensen [13] advancing nursing practice definition. Christensen integrated Benner's clinical competency model and Rolfe's knowledge typology theory to describe advancing nursing practice. According to Christensen [13] advancing nursing practice is professional development process of knowledge and skills acquisition to reach to proficiency in clinical practice. Further illustration of advancing nursing practice is presented in the literature review section. There is an increasing amount of evidence supporting the notion that the more advanced knowledge and skills a nurse has pertaining to his or her area of specialty, the better his or her patients' outcomes [14]. The researcher studied the lived experiences of cardiac nurses working to advance their practice using online (CPD) activities to yield deep insight into the effectiveness of this learning mode [15-17].
The hermeneutic phenomenological perspective influenced the interview process by using a systematic approach to the phenomenological interview. This manuscript provides a description of the study setting and study sample. Also, this study explains the process of gathering rich, indepth data using one to one unstructured interview.

\section{Methods:}

Phenomenology hermeneutic is the philosophical framework that guided this study. This relativist ontological, emic epistemological approach was used to achieve in-depth understanding of the nurse experience of learning using an online CPD program as a tool to advance their practice. This methodology uncovers the lived experience to reveal meanings embedded within the lived experience and uses an interpretive process to increase understanding of these meanings. Therefore, data gathering techniques in this study focused on gaining deep information by exploring the nurse experience.

\section{Study setting}

The study was conducted in one of the largest accredited teaching hospitals in South Australia. This hospital provides many cardiac services for patients from South Australia, the Northern Territory, and South East Asia who are referred from the AUS-health services. The cardiology department is divided into two main units: the cardiology unit which provides medical and interventional cardiac services and the cardiothoracic unit which provides surgical services. The department is staffed by a specialised multi-professional team, some of whom have completed specialised cardiac post-graduation diploma courses in order to meet the complex needs of cardiac patients. To keep the team abreast of the latest developments in practice, the in-service education department at local study setting runs several continuing professional development (CPD) programs for the cardiology department via varied learning modes, such as online CPD programs, classroom learning and bedside-based learning. The nursing team maintains advanced clinical skills through online CPD, orientation programs, and in-service classroom-based courses. Regarding online CPD courses, electrocardiography interpretation and underwater sealing draining management courses are mandatory courses which all registered nurses must complete while working in medical or surgical cardiac wards.

\section{Population}

Purposive sampling was used to select participants because the researcher aimed to recruit nurses able to reflect on the experience of advancing their skills in the specialised area of cardiology. According to Schneider, Whitehead [18], purposive sampling is appropriate when the researcher wants to collect rich, in-depth information from participants who have special knowledge or experience that the researcher is looking.

The target sample was registered nurses without speciality qualifications with more than one year experience working in medical and surgical cardiac wards. As the aim of phenomenological study is to develop rich and deep information about the phenomena, the sample size was determined using the principle of saturation. Data saturation is repetition of discovered data and confirmation of previously collected data [19]. The aim of data saturation is to gather a large pool of rich and deep information and to ensure that all data about the phenomena under investigation has been collected, whereby the researcher can extract new themes for the phenomena [19]. The researcher reaches the point of data saturation when no further new information is obtained [20]. In this study, data saturation was achieved with five nurses.

The purposive sample was selected from an accessible population representing the specialised area of one hospital. A reason for choice of this specific hospital was that it is a tertiary hospital where most of the departments are specialised and have high patient acuity, so the researcher 
was able to find the target sample. As pointed out by Fain [21], the researcher must choose an accessible population that represents the target sample.

\section{Inclusion and exclusion criteria}

In phenomenological research, the participants are expected to reflect upon their experience for the intended topic. Therefore, inclusion and exclusion criteria must be carefully established [18].

\section{Inclusion}

The sample was comprised of registered nurses (RNs), beyond their firstyear post graduation, who do not have a specialty qualification and are working in medical and surgical cardiology. This target group was included in the study because of the unique nature of work in the cardiology setting. More specifically, compared with patients in a general clinical setting, the condition of cardiac patients is more likely to change rapidly, which requires nurses with advanced knowledge and skills to make appropriate and timely decisions.

\section{Exclusion}

Participants excluded from the study includeds registered nurses who work in other departments, and ward managers and nursing supervisors of departments in the selected setting. The rationale for not including such participants is because the researcher wanted to explore the perceptions of nurses who have daily patient contact which requires them to possess a high level of competency. According to Oman, Fink [22] exclusion criteria enables the author to get the required sample and valid findings.

Newly graduated nurses with less than one-year experience was excluded because they are not required to practice specialised nursing skills in the first year after graduation. Nurses with nursing post-graduate certification in cardiology were also excluded because they already have gained competencies and skills via the post-graduation cardiology course, and their learning needs are more advanced than those of other nurses.

\section{Recruitment}

The researcher used a three-stage recruitment strategy. The first stage of introduction sessions was conducted to explain the objectives and significance of the research to nurses working in the medical and surgical cardiology wards. After the session, the researcher distributed a participant information sheet which explained participation procedures and listed the researcher's contact details (see appendix 1).

Also, the researcher distributed recruitment information flyer in the cardiology words (see appendix 2). Through conducting this stage, the researcher ensured that potential participants understood the inclusion criteria of the study and were able to contact the researcher if they decided to volunteer to participate. In the second stage, the researcher contacted volunteer participants to arrange an interview day and time. In the third stage, the researcher met volunteer participants on the scheduled day of interview and provided them with a consent form to sign before each interview commenced (see appendix 3). Also, the researcher used the snowball recruitment strategy as the participation level was proven to be too low for data saturation. Snowball sampling is the use of one participant to source the next participant and is a useful strategy when there is difficulty recruiting participants [19].

\section{Data collection tool}

The researcher conducted an unstructured interview with each participant to explore the nurse experience with online CPD. Interviews took from 30 minutes to one hour. The rationale of choosing this tool was because the researcher was able to format discussion questions that covered all study objectives. Also, the researcher was able to ask participants to elaborate in order to clarify a point or obtain more information. According to Schneider, Whitehead [18], interviews give the researcher many privileges, such as the opportunity to gather deep, detailed information and clarify information from participants. The researcher used several tactics during the interview that added richness to collected data. Further explanation about the data collection tool is discussed in the interview process section.

\section{Ethical consideration}

Ethical approval

The researcher obtained approval from the Royal Adelaide Hospital Human Research Ethics Committee. Ethical approval was granted and given number HREC/16/RAH/168) (see appendix 4).

\section{Informed consent}

The researcher obtained written informed consent from participants to ensure that autonomy was maintained. The researcher is responsible to ensure that participants understand the study, including benefits, risks, and participant rights [21]. Thus, participants were provided with information sheet along with the consent form (see appendix $\mathbf{2 \& 3}$ ). The participant information sheet outlined the purpose of study, procedures, risks, benefits, and participant rights. Also, it included the ethical committee approval code and the ethical committee contact number to call if they had concerns regarding the data collection process. The researcher ensured that participants read and understood the information sheet before giving written consent. According to Fain [21], informed consent is one of the procedures that can be used to protect participant rights.

\section{Anonymity and confidentiality}

In interview it is difficult to maintain participant privacy and confidentiality due to face to face interaction and the depth of information which may easily reflect participant identity [19]. However, the researcher in this study used the following strategies to protect participant confidentiality and anonymity. First, the researcher offered participants interview venue away from their clinical area, so their colleagues and ward manager were not aware they were being interviewed. However, participants preferred to have the interview conducted in a quiet private room in their workplace, ensuring that the room was not recognised as an interview room and they did not inform others they had been interviewed. Second, the researcher guaranteed that no one would have access to the primary source of data except the researcher. Third, the researcher respected the right of participants to withdraw from the study at any time and ensured that they were aware about this right before giving signing the consent form. Fourth, the researcher used pseudonyms on interview transcripts and transcripts were not shown to anyone other than the researcher and supervisors and they are saved in locked file so no one else can access them. Finally, the researcher numbered the interview recording tapes, so they were not carrying participant names with the list of names stored separately in a password processed file. The password was shared with research supervisors.

\section{Data storage}

The researcher safely stored the recorded and the transcribed interviews; this data did not contain the participants' names. The list of participants' names was stored in a password-protected file. The files and passwords were shared only with the research supervisors.

\section{Data collection}

Data collection in phenomenological hermeneutic research involves gathering of participant experiences and related reflections to explore the deep and significant meanings of their experiences [23]. van Manen [23] listed several types of tools to serve the purpose of phenomenological hermeneutic research, such as interviewing and observation. Experiential 
anecdotes and experiential descriptions found in the literature, biographies, diaries, and journals are part of phenomenological data [23].

\section{Results:}

The interview process was conducted in five stages as described below.

\section{Step one: Determining the type of the interview}

van Manen [23] argued that in phenomenological hermeneutic research, the method does not rule the question, but the question determines the method appropriate to build up the direction which provides an understanding of a human phenomenon. Therefore, in such types of qualitative studies the researcher should focus on the fundamental question of the phenomenological inquiry throughout the unstructured, indepth interview process.

\section{Step two: Making initial contact}

The researcher established direct contact with each participant prior to the interview day. By doing so, the researcher established a rapport with the participant and prepared them mentally by giving them the participant information sheet. van Manen [24] encouraged researchers to develop openness and closeness with the participant to gain their trust before conducting a phenomenological interview, especially if the interview is around their personal experience, something which is not easily disclosed by everyone.

\section{Step three: Context of the interview}

All interviews were conducted in a quiet room in the ward. Although it was proposed that interviews be conducted in a quiet room in the School of Nursing in order to maintain participant privacy and anonymity, participants requested to conduct the interviews in their work setting. Nonetheless, the researcher ensured that participant privacy and anonymity was upheld. In fact, van Manen [24] suggested that a phenomenological interview should be conducted in a place where the surrounding atmosphere triggers interviewees to recall their experience. Participants in this research were able to recall some examples of the cases which they cared for and applied the knowledge gained from the online CPD ECG interpretation course or the online under water sealing chest draining course.

\section{Step four: Selecting the lived experience}

Each participant was interviewed once. Interview duration was 15 to 30 minutes. The interviews started with a grand tour question (see appendix 5). Grand tour questions are very broad questions asked by the interviewer at the early stage of an interview to obtain a description of the event or experience [25]. This type of question gives participants freedom to express their lived experience. Furthermore, the researcher used some other interview tactics to control the interview process and focus on the aim of the interview, such as probing and rephrasing questions (see appendix 5). The researcher used direct and indirect probing to encourage participants to elaborate more on the issues they were expressing (see appendix 5). Guest [25] stated that probing involves inductive and unscripted questions based on the last response of an interviewee. The researcher also used probing to steer the conversation during the interview when participants diverted from the main topic. Probing is a key element in a successful unstructured in-depth interview because it helps one to pull more inductive thoughts from participants to promote rich and deep responses [25].

Guest [25] argued that the interviewee is the expert of their own experience and opinions, so it is important to grasp their meaning accurately. The researcher rephrased some of the thoughts and sentences that participants expressed to ensure what the researcher understood was correct. Participants tended to give examples when the researcher rephrased their words for further understanding, and this was helpful to gaining deeper information.

The researcher used two audio recorders during interview in case one failed. Recording the interview allow the researcher to fully engaged with participant descriptions of their experience. Also, the researcher was able to take memos of nonverbal expressions, words, and statements that helped during the interpretation and data analysis stages.

Guest (2013) [26] stressed maintaining consistency across the interview by using tactics to gather the optimal data required. The researcher used prepared notes during the interview in order to focus on the phenomenon in question. The prepared notes contained some keywords related to presumptions about the phenomenon. van Manen [24] encouraged researchers conducting phenomenological research to be prepared mentally for an interview and focus on the main aim of the interview and obtain concrete data about participant experiences, stories, and examples. In this study, the researcher did not allow presumptions to impact on participant thoughts and descriptions.

\section{Step five: Concluding the interview}

All interviews were concluded by thanking the participant and offering them the choice to have a copy of their interview transcript to verify what they had said. The researcher wrote an interview summary after listening to the interviews on the same day. The summary was prepared to help the researcher evaluate the amount of data gathered and identify whether the point of data saturation was reached. In addition, writing the summary helped the researcher reflect on the interview and gain an understanding of the participant experience.

\section{Discussion:}

The essential themes and essence of the lived experience can be grasped by textual reflection on lived experience. van Manen [23] emphasised that a phenomenological researcher should be totally engaged with textual reflection as it is the only way to approach the meaning of human experience. Also, he stressed that a researcher should be thinking of the phenomenon described in the text while approaching the intended unity of meanings, meaning structures, or themes.

The researcher conducted unstructured interviews with participants to explore their experience with online CPD. van Manen [23] emphasised that interviewing is an appropriate method for phenomenological hermeneutic research as it provides a narrative of the participant experience, thus ensuring rich and deep understanding. In fact, van Manen [23] stated that this type of interview aims to gather experiential material, such as stories, anecdotes, recollection of experiences and reflection on those experiences. In other words, data collection is a tool for gathering lived experience materials which are beyond subjective data collection tools of other qualitative research such as observation in ethnography study. In order to achieve the purpose of the interview, the researcher must be oriented to the phenomenological question of "what is the nature of the phenomenon' (i.e. the contribution of online CPD to nursing skills advancement) [23]. To further understand how phenomenological questions interplay during data collection, van Manen [23] produced six activities to be followed during data collection which are described in the following section. van Manen [24] defined method as a way or attitude that directs the researcher to understand the phenomenon.

The phenomenon of interest stemmed from the researcher's own experience of using e-learning material as a learning approach for skills advancement. As the researcher's background is cardiology nursing, the concern was around correlation between the use of available online CPD materials and cardiac nursing skills advancement. The researcher wondered what the experiences of other registered nurses were when they used this type of learning approach. Did they have a similar experience as 
the researcher? How did they perceive these courses in advancing their clinical skills? What meanings do such experiences add to their life in general and in professional terms, in particular? At this stage, the researcher became oriented to the phenomenon of interest and started exploring the nature of this phenomenon through others' experiences. This means the researcher's reflection and pre-understanding of the experience is the foundation of a phenomenological question. van Manen [23] claimed that being oriented to the phenomenon means the essential nature of a person's lived experience is sensed and the researcher raises phenomenological questions and concerns.

Investigating the experience as it is lived starts with gathering experiential materials, such as participant stories, experience descriptions, and experience reflections that can lead to exploration of the fundamental nature and not the conceptuality of the lived experience [23]. In this study, the researcher started with her own personal experience using the online CPD program for skills and knowledge enhancement. van Manen [23] stresses, the description of the researcher's own personal experience gives a basic idea of the phenomenon which helps in being oriented with the main question through all stages of the research. As it is a lived experience that the researcher is investigating, experiential materials were gathered in textual, dialogical, and conversational forms.

As pointed out earlier, the researcher used unstructured, open-ended interviews to gather participant experiential accounts. More specifically, the phenomenological interview principles of van Manen [24] were applied. van Manen [24] differentiated between phenomenological and hermeneutic interviews stating that a phenomenological interview is a tool to gather experiential materials, whereas a hermeneutic interview is a tool to purely interpret data gained from a phenomenological interview, observation, or other means of data collection [24]. The reason behind not choosing the hermeneutic interview for this research is that the researcher was not expecting participants to interpret their own experiences. In fact, [24] highlighted the failure of people to accurately interpret their own experiences.

In phenomenological hermeneutic research, the purpose of transcribing an interview is to capture the participants' own words and at the same time giving privilege to the researcher to interpret those words. The researcher should ensure that the transcription accurately reflects the reality of the experience. Errors during the interview transcribing, such mishearing or misinterpretation of words, can change the meaning of the phrase, lead to missing some key themes, or bring up non-existent themes [27]. In this study, the researcher transcribed interviews verbatim, producing textual accounts of the participant experience from which the essential themes and meanings were extracted. Transcription was written in dialogical conversational form, presenting the interview as it had been conducted without reduction. To avoid errors and misinterpretation during transcription in this research, the researcher listened to the interview recordings three times: [1] without transcribing to grasp the full experience, [3] listening and transcribing, and [3] listening to verify the transcript. The researcher offered the participants their interview transcripts for further verification.

Though the researcher ensured that the transcription was accurate, the meaning within the text can be self-alienated after transcription [23]. In other words, to apply textual reflection, the researcher has to consider a process that transforms the text to its real meanings. The hermeneutic circle introduced by Gadarmer was used to bring out the meaning e buried within the text during transcription. Text interpretation is elaborated upon further in the interpretation section.

Understanding the art of writing and rewriting is essential in phenomenological research. van Manen [23] stressed that language is the only way for textual symbolic form. As indicated earlier, phenomenological research aims to transform the lived experience into a textual expression in which the text reflects meaning. van Manen [23] stated that creating phenomenological text is part of the research process and pointed out that writing is fused with research and the text has to reflect itself. This means the process of writing is more than just a demonstration of information. Hence, during data collection in this study, the researcher was very conscious of verbal and nonverbal language used by participants.

The researcher was mindful of the phenomenological inquiry through the interview process to prevent participants from diverting from the main topic. However, some participants lost focus and started talking about their colleagues' experiences with online CPD programmes. However, the researcher was able to bring the conversation back to the main topic by rephrasing the question and referring to their experience using some keywords the participant had mentioned about themselves. Being strongly oriented to the phenomenon helps prevent the rise of superficialities in the description of the lived experiences [23].

To balance the research context means to constantly measure the whole text against the significant part that influences the meaning of the whole textual structure [23]. To achieve balance in context during the data collection phase, the researcher implemented two strategies. First, the researcher was engaged in the study of the fundamental phenomenological question through the interview process. Second, frequent dealing with audio taped interviews and transcripts, and creating summaries and memos, helped capture the experience within the context. van Manen [23], however, argued that being so immersed in phenomenological inquiry may lead the researcher to lose the perspective of the dialogical text which contains deep meanings of the phenomenon. Therefore, the researcher ensured that the balance of context was achieved by adopting a cycle that started with data collection, followed by thematic analysis and interpretation. This cycle enabled the researcher to determine whether themes extracted from interview transcripts reflected the context and phenomenon overall.

\section{Conclusion}

This study explained the process of data collection, describing the setting, nature of participants and process of data collection using phenomenological interview. As the human experience is complex, gathering in-depth data should be systematic to ensure that the researcher has obtained the most sufficient data to explore the essence of the experience.

Conflict of Interest (COI) statement: "The authors declare no conflicts of interest"

\section{Financial Disclosure statement: None}

\section{References}

1. Al Salmi I, Hannawi S. The World Health Report-Health systems Empowering Citizens and Improving Performance. Research on Humanities and Social Sciences. 2016;6(2):6.

2. Al Salmi I, Hannawi S. Health Workforce in the Sultanate of Oman: Improving performance and the Health System. J Int Med Pat Care. 2018;1(1):6.

3. Hannawi S, Al Salmi I. Health workforce in the United Arab Emirates: analytic point of view. Int J Health Plann Manage. 2014;29(4):332-341.

4. Hannawi S, Al Salmi I. Time to address gender inequalities against female physicians. Int $\mathbf{J}$ Health Plann Manage. 2018;33(2):532-541.

5. Cooper E, Spilsbury K, McCaughan D, Thompson C, Butterworth T, Hanratty B. Priorities for the professional development of registered nurses in nursing homes: a Delphi study. Age Ageing. 2017;46(1):39-45. 
6. Haywood H, Pain H, Ryan S, Adams J. The continuing professional development for nurses and allied health professionals working within musculoskeletal services: a national UK survey. Musculoskeletal Care. 2013;11(2):63-70.

7. Neubeck L, Lin SH, Ferry C, Gallagher R. What does the Development of the European Core Curriculum for Cardiovascular Nurses Mean for Australia? Heart Lung Circ. 2016;25(4):407-409.

8. Gould D, Papadopoulos I, Kelly D. Tutors' opinions of suitability of online learning programmes in continuing professional development for midwives. Nurse Educ Today. 2014;34(4):613618.

9. Wakefield PL, Wilson MA. Enhancing nurses' knowledge regarding the complex care of hospitalized patients on insulin. J Nurses Prof Dev. 2014;30(4):174-180.

10. Edmonds M, Adams J. Evaluating the impact of a cardiac module for post-registration nurses. Nursing Standard. 2009;24(2):35-41

11. Riley J, Brodie L, Shuldham C. Cardiac nursing: Achieving Competent Practitioners. European Journal of Cardiovascular Nursing. 2005;4(1):15-21.

12. Boyde M, Jen C, Henderson A, Winch S. A clinical development unit in cardiology: the way forward. International Journal of Nursing Practice. 2005;11(3):134-139

13. Christensen M. Advancing practice in critical care: a model of knowledge integration. Nursing in Critical Care. 2009;14(2):8694.

14. Riley J, Brodie L, Shuldham C. Cardiac nursing: achieving competent practitioners. Eur J Cardiovasc Nurs. 2005;4(1):15-21.

15. Carroll C, Booth A, Papaioannou D, Sutton A, Wong R. UK health-care professionals' experience of on-line learning techniques: a systematic review of qualitative data. J Contin Educ Health Prof. 2009;29(4):235-241.

16. Feldacker C, Jacob S, Chung MH, Nartker A, Kim HN. Experiences and perceptions of online continuing professional development among clinicians in sub-Saharan Africa. Hum Resour Health. 2017;15(1):89.

17. Underhill ML, Dickerson SS. Engaging in medical vigilance: understanding the personal meaning of breast surveillance. Oncol Nurs Forum. 2011;38(6):686-694.

18. Schneider Z, Whitehead D, LoBiondo-Wood G, Haber J. Nursing and midwifery research : methods and appraisal for evidencebased practice. 4th ed. Sydney: Mosby Elsevier; 2013.

19. Streubert H, Carpenter D. Qualitative research in nursing : advancing the humanistic imperative. 5th ed. Philadelphia: Wolters Kluwer Health/Lippincott Williams \& Wilkins; 2011.

20. Polit DF, Beck C. Nursing Research: generating and assessing evidence for nursing practice. 8th ed ed. Philadelphia: Wolters Kluwer / Lippincott Williams \& Wilkins; 2008.

21. Fain A. Reading, Understanding, and Applying Nursing Research. 4th ed. Philadelphia: F.A.Davies; 2014.

22. Oman k, Fink R, Krugman M. Nursing Research Secrets. London: Elsevier Health Science; 2003.

23. van Manen M. Researching lived experience : Human science for an action sensitive pedagogy 2 nd ed. London: Althouse Press; 1997.

24. van Manen M. Phenomenology of Practice. California: Left Coast Press; 2014.

25. Guest G. Collecting qualitative data : a field manual for applied research. Namey EE, Mitchell ML, editors. Los Angeles: SAGE; 2013.

26. Guest EM, Keatinge DR, Reed J, Johnson KR, Higgins HM, Greig J. Implementing and evaluating a professional practice framework in child and family health nursing: a pilot project. Nurse Educ Pract. 2013;13(5):393-399.

27. Easton KL, McComish JF, Greenberg R. Avoiding common pitfalls in qualitative data collection and transcription. Qualitative health research. 2000;10(5):703-707.

\section{Appendix 1: Participants information sheet \\ PARTICIPANT INFORMATION SHEET}

PROJECT TITLE: The lived experiences of nurses working in cardiology services with online continuing professional development programs to advance their specialised clinical practice: A phenomenological study.

Dear Participant,

You are invited to participate in the research project described below.

\section{What is the project about?}

The research is about exploring the registered nurses experience with online continuing professional development programmes in advancing their cardiac knowledge and skills. This research will help to understand the experience of bedside registered nurses without specialised qualifications, in seeking to advance their clinical skills by undertaking online continuing professional development (CPD).

\section{Who is undertaking the project?}

This research will be undertaken by Umaima Al-Raisi as a part of Master degree programme in Nursing Science at the University of Adelaide under the supervision of:

Associate Professor Judy Magarey, Deputy Head of School (Learning and Teaching)

School of Nursing

The University of Adelaide

\section{Dr Philippa Rasmussen}

Master of Nursing Science Program Coordinator

School of Nursing

The University of Adelaide

\section{Why am I being invited to participate?}

You are invited to participate in this research because you are registered nurse, have more than one year of experience, do not have the speciality qualification and you are working in medical or surgical cardiology.

\section{What will I be asked to do?}

You will be asked to participate in a semi structured interview and talk about your experience with online continuing professional development programs. The interview will be conducted in a private room in the school of nursing at the University of Adelaide. The interview will take approximately 30 to 60 minutes.

Each participant will be interviewed individually and it will be audio recorded. Confidentiality will be maintained; your identity will not be disclosed.

\section{Are there any risks associated with participating in this project?}


No risks have been identified for you in this research. However, you can stop the interview or withdraw from the study at any time if you feel distressed and uncomfortable while talking about your experience.

\section{What are the benefits of the research project?}

There is no personal benefit gained from this research. However, understanding your experience will help guide nursing leaders and educators to develop and evaluate a system that will promote online continuing professional learning among staff nurses. The finding of the study may inform and changed required to the mandatory online of continuous professional activities to serve the need of the specialised settings.

\section{Can I withdraw from the project?}

Participation in this research is voluntary. You can withdraw at any time of the study without any consequence.

\section{What will happen to my information?}

The researcher will ensure that your confidentiality and anonymity is be maintained through: using pseudonyms on the interview transcripts and code number on the recorded tapes. The transcripts and the list of names will be stored separately in a password processed file. The password will only be shared with the researcher's supervisors. The data collected will only be used for the study purposes and your personal information will not be identified in the masters thesis or any other publication.

\section{Who do I contact if I have questions about the project?}

For further details or questions please contact:

Primary researcher:

\section{Umaima Al-Raisi}

Student in Master of Nursing Science (Cardiology), the University of Adelaide.

Mobile No (Australia): +61450840757

Mobil No (Oman): +9689900911

E-mail: umaimamahmoodabdul.alraisi@student.adelaide.edu.au

Research Supervisors:

Associate Professor Judy Magarey
Office No: +61883133594

Email: judy.magarey@adelaide.edu.au

\section{Dr Philippa Rasmussen}

Office No: +61 882222991

Email: philippa.rasmussen@adelaide.edu.au

\section{What if I have a complaint or any concerns?}

The study has been approved by the Research Ethics Committee at the Royal Adelaide Hospital (approval number HREC/16/RAH/168). The research will be conducted according to the NHMRC National Statement on Ethical Conduct in Human Research, 2007. If you have questions or problems associated with the practical aspects of your participation in the project, or wish to raise a concern or complaint about the project, and then you should consult the Principal Investigator.

If you wish to speak to someone not directly involved in the study about your rights as a volunteer, or about the conduct of the study, you may also contact the Chairperson, Research Ethics Committee, and Royal Adelaide Hospital on 82224139.

\section{If I want to participate, what do I do?}

If you fit the inclusion criteria and you wish to participate, you need to contact the primary researcher on the mentioned above contact details via a call, text message, or an email. Then you will receive a call from the researcher to schedule a day for an interview. Before starting the interview, the researcher will ask you to read the information sheet and then if you choose to participate sign a written consent. By signing the consent, you show your:

Understanding about the consent statement.

Willingness to be part of the research.

Understanding about the research as described in the participant sheet.

Yours sincerely,

Umaima Al-Raisi

Student in Master of Nursing Science (Cardiology)

School of Nursing

The University of Adelaide

\section{Appendix 2: Recruitment information flyer}




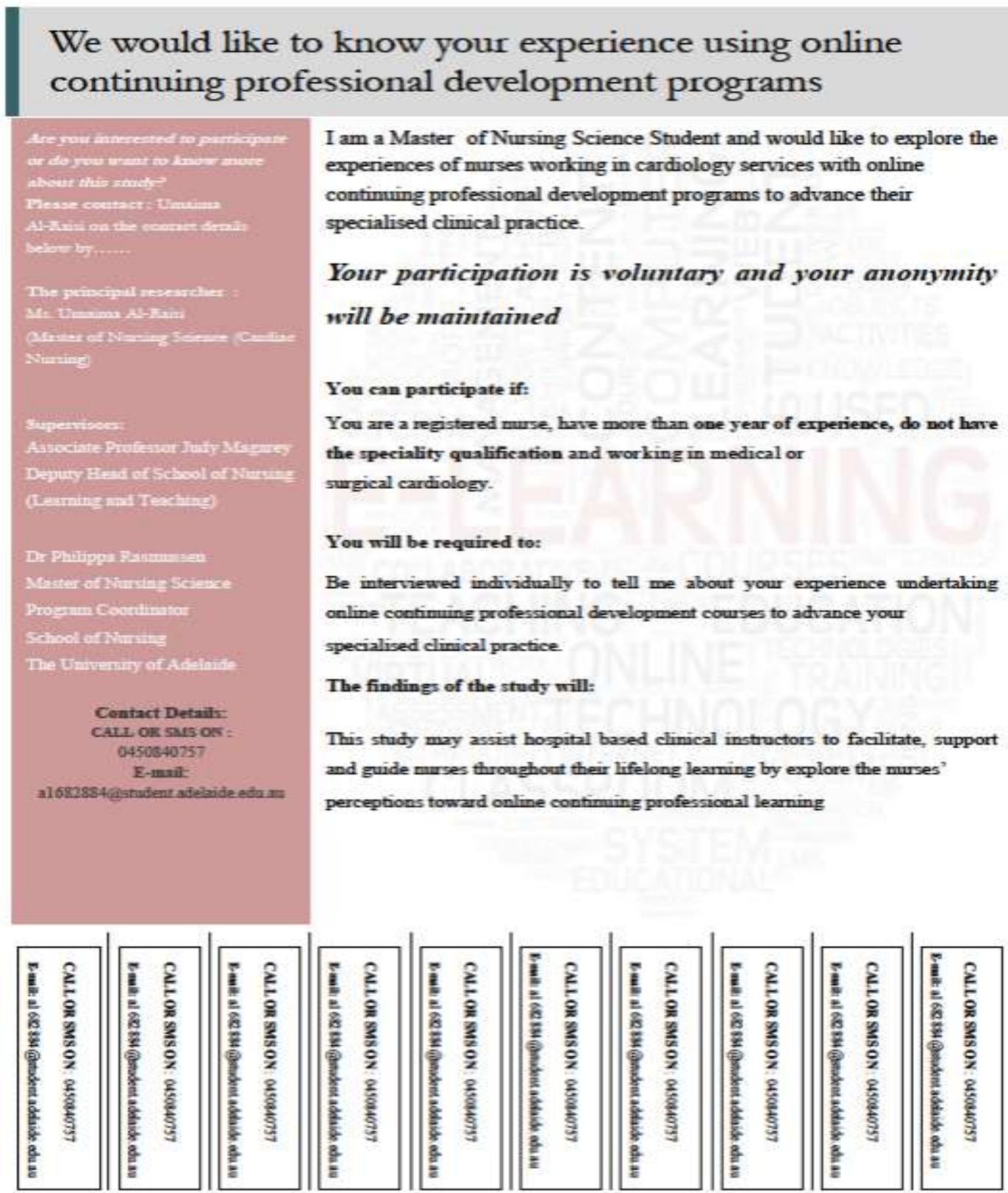

Appendix 3: Consent form

\section{Consent form}

PROTOCOL NAME: The lived experiences of nurses working in cardiology services with online continuing professional development programs to advance their specialised clinical practice: A phenomenological study.

\section{INVESTIGATORS:}

This research will be undertaken by Umaima Al-Raisi as a part of Master of Nursing Science program at the University of Adelaide under the supervision of:

Assoc. Prof. Judy Magarey, RN, BNurs, MNurs (research), DNurs.

Dr Philippa Rasmussen, RN BN, Grad Cert CAMHN, Grad Dip Psychological Studies, MNurs, PhD.

Thereby, I certify that:
- $\quad$ I read and understood the participant information sheet, and the researcher has answered my queries and I understand the purpose, procedures and risks of the study.

- I I understand that I will not financially benefit from taking part of the study.

- I understand my participation is voluntary and I can withdraw at any time.

- I understand that I may stop the interview at any time if I feel uncomfortable or I do not want to continue and any written or recorded information provided by me will not be included in the study.

- I I understand that I may refuse to answer any question if I wish. 
- I understand that copies of the transcripts may be return to me for verification.

Name of the subject:
Signed:

Dated:

I certify that I have explained the study to the participant and consider he/ she understand what is involved:

Signed:

Dated:

\section{Appendix 4: Ethical Approval}

\section{Approval Date: 02 May 2016}

HREC Reference number. HREC/16/RAH/168

CALHN Reference number: R20160435

Ms Umaima Mahmood Al-Raisi

School of Nursing

UNIVESITY OF ADELAIDE

\section{Dear Ms Mahmood Al-Raisia}

Project Title: The lived experiences of nurses working in cardiology services with online continuing professional development programs to advance their specialised clinical practice: a phenomenological study

Thank you for submitting the above project for ethical review. This project was considered by the Chairman of the Royal Adelaide Hospital Human Research Ethics Committee. I am pleased to advise that your protocol has been granted full ethics approval and meets the requirements of the National Statement on Ethical Conduct in Human Research, incorporating all updates. The documents reviewed and approved include:

- Study protocol

- Participant Information Sheet

- Participant Consent Form

- Advertisement

\section{GENERAL TERMS AND CONDITIONS OF ETHICAL APPROVAL:}

- Adequate record-keeping is important. If the project involves signed consent, you should retain the completed consent forms which relate to this project and a list of all those participating in the project, to enable contact with them in the future if necessary. The duration of record retention for all clinical research data is 15 years.

- You must notify the Research Ethics Committee of any events which might warrant review of the approval or which warrant new information being presented to research participants, including:

(a) serious or unexpected adverse events which warrant protocol change or notification to research participants.

(b) changes to the protocol,

(c) premature termination of the study.

- The Committee must be notified within 72 hours of any serious adverse event occurring at this site.

- Approval is valid for $\mathbf{5}$ years from the date of this letter, after which an extension must be applied for. Investigators are responsible for providing an annual review to the RAH REC Executive Officer each anniversary of the above approval date, within 10 workings days, using the Annual Review Form available at: hitto://www rah.sa.gov.au/reclindex.php

- The REC must be advised with a report or in writing within 30 days of completion.

Should you have any queries about the HREC's consideration of your project, please contact Ms Heather O'Dea on 0882224139 , or Health. CALHNResearchEthics_sa.gov.au.

You are reminded that this letter constitutes ethical approval only. You must not commence this research project at a SA Health site until governance authorisation from the Chief Executive or delegate of that site has been obtained.

This Committee is constituted in accordance with the NHMRC's National Statement on the Ethical Conduct of Human Research (2007).

The HREC wishes you every success in your research.

Yours sincerely.

\section{AVProf A Thornton}

CHAIRMAN

RESEARCH ETHICS COMMITTEE 


\section{Appendix 5: Sample interview questions \\ Objective of the study}

To understand the experience of bedside registered nurses without specialised qualifications, in seeking to advance their clinical skills by undertaking online continuing professional development (CPD).

\section{Open ended questions}

As you it is explained in the participant information sheet, this study about exploring your experience with using online CPD course and it relation in advancing your clinical skills and knowledge in your working area.

- So first I would like to know which courses you were enrolled in?

- What you would like to tell me about your experience?

- How do you describe your experience using this course for example: the ECG interpretation course?

- How do you perceive your experience using the online courses for your professional development and skills enhancement?

Rephrasing participant's words
- $\quad$ you said that the course is good, can you explain more what do you mean by good?

- You mentioned that mentors are important, can you elaborate more why you think they are important?

- You just said that the course is vague? How it is vague? Can you explain that?

- you mentioned that it is good to have someone to discuss with, can you tell me why?

\section{Direct and indirect probing}

Direct probing by repeating some words to encourage the participant to continue describing their experience:

- Yes

- really!

- that sound interesting

- $\operatorname{good}$

- great,

- that sound exciting Indirect probing by: nodding head and sustain eye contact with the participants

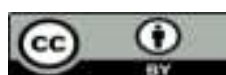

This work is licensed under Creative Commons Attribution 4.0 License

To Submit Your Article Click Here: Submit Article

DOI:10.31579/2641-0419/102
Ready to submit your research? Choose Auctores and benefit from:

* fast, convenient online submission

* rigorous peer review by experienced research in your field

* rapid publication on acceptance

* authors retain copyrights

* unique DOI for all articles

* immediate, unrestricted online access

At Auctores, research is always in progress.

Learn more www.auctoresonline.org/journals/clinical-cardiology-andcardiovascular-interventions- 